\title{
Dynamical quantum phase transitions and the Loschmidt echo: A transfer matrix approach
}

\author{
F. Andraschko and J. Sirker \\ Department of Physics and Research Center OPTIMAS, \\ Technical University Kaiserslautern, D-67663 Kaiserslautern, Germany
}

(Dated: June 7, 2018)

\begin{abstract}
A boundary transfer matrix formulation allows to calculate the Loschmidt echo for onedimensional quantum systems in the thermodynamic limit. We show that nonanalyticities in the Loschmidt echo and zeros for the Loschmidt amplitude in the complex plane (Fisher zeros) are caused by a crossing of eigenvalues in the spectrum of the transfer matrix. Using a density-matrix renormalization group algorithm applied to these transfer matrices we numerically investigate the Loschmidt echo and the Fisher zeros for quantum quenches in the XXZ model with a uniform and a staggered magnetic field. We give examples - both in the integrable and the nonintegrable cases - where the Loschmidt echo does not show nonanalyticities although the quench leads across an equilibrium phase transition, and examples where nonanalyticities appear for quenches within the same phase. For a quench to the free fermion point, we analytically show that the Fisher zeros sensitively depend on the initial state and can lie exactly on the real axis already for finite system size. Furthermore, we use bosonization to analyze our numerical results for quenches within the Luttinger liquid phase.
\end{abstract}

PACS numbers: 05.70.Ln,64.70.Tg,75.10.Pq

\section{INTRODUCTION}

The time evolution of a quantum system is governed by a unitary operator $U(t)$. As a consequence, the evolution of a quantum state in time, $|\Psi(t)\rangle=U(t)\left|\Psi_{0}\right\rangle$, where $\left|\Psi_{0}\right\rangle$ is the initial state, can be reversed $\left|\Psi_{0}\right\rangle=$ $U^{\dagger}(t)|\Psi(t)\rangle$. The question how stable such a time reversal is with respect to small perturbations is of fundamental importance to understand decoherence, which leads to the emergence of classical behavior and sets limits for quantum computation. ${ }^{[12}$ For a many-body system, one might expect on general grounds an inherent instability related to the dynamical complexity of the problem and quantum chaos $\frac{\sqrt[3]{5}}{5}$

A measure of the stability of time reversal is the Loschmidt echo defined by ${ }^{6}$

$$
\mathcal{L}(t)=\left|\left\langle\Psi_{0}\left|e^{i H\left(g_{1}\right) t} e^{-i H\left(g_{0}\right) t}\right| \Psi_{0}\right\rangle\right|^{2} .
$$

Here, $H(g)$ is a time-independent Hamiltonian which is a function of a microscopic parameter $g$ whose value is assumed to change from $g_{0}$ during the forward time evolution to $g_{1}$ during the backward time evolution. $\mathcal{L}(t)$ thus is nothing but the fidelity between the state time-evolved with $H\left(g_{0}\right)$ and the state time-evolved with $H\left(g_{1}\right){ }^{[7} \mathrm{An}$ advantage of the Loschmidt echo in comparison to many other quantum information measures is that it is accessible experimentally, for example, by nuclear magnetic resonance. $\stackrel{89}{ }$

In this paper, we are interested in the recent idea to use the Loschmidt echo to study and classify quenches in closed quantum systems. We concentrate on the case where $\left|\Psi_{0}\right\rangle$ is the ground state of $H\left(g_{1}\right)$. In this case, the Loschmidt echo reduces to

$$
L(t)=\left|\left\langle\Psi_{0}\left|e^{-i H t}\right| \Psi_{0}\right\rangle\right|^{2},
$$

where we have set $H \equiv H\left(g_{0}\right)$. The Loschmidt echo can be calculated exactly for bilinear Hamiltonians such as the transverse Ising model. ${ }^{10111}$ In Ref.11, it has been, furthermore, pointed out that the Loschmidt amplitude $Z(z)$ has the form of a partition function with boundaries fixed by the initial state,

$$
Z(z)=\left\langle\Psi_{0}\left|e^{-z H}\right| \Psi_{0}\right\rangle,
$$

with $z \in \mathbb{C}$. Here, a general complex parameter $z$ allows to investigate the analytic structure of $Z(z)$ in the whole complex plane.

The motivation for such an approach are studies of the analytic properties of the thermodynamic grand canonical partition function $Z_{\mathrm{th}}(\beta, \mu)=\operatorname{Tr} \mathrm{e}^{-\beta(H-\mu N)}$ for complex fugacity $\tilde{z}=\exp (\beta \mu)$ ( $\beta$ is real) or of the canonical partition function $Z_{\mathrm{th}}(\beta)=\operatorname{Tr} \mathrm{e}^{-\beta H}$ for complex inverse temperature $\beta$, which have helped to understand and classify phase transitions ${ }^{[12}$ For $\beta$ and $\tilde{z}$ real, the grand canonical partition function is a sum of positive terms and thus strictly positive. Furthermore, $Z_{t h}$ is a sum of analytic terms and therefore an analytic function for any finite system size. Phase transitions, signalled by nonanalyticities in the corresponding thermodynamic potential, can therefore only occur in the thermodynamic limit. In this limit, zeros in the complex $\tilde{z}$ or complex $\beta$ plane will close in on the real axis at the transition point so that the thermodynamic potential becomes nonanalytic.

The grand canonical partition function $Z_{\mathrm{th}}(\beta, \mu)$ is a polynomial in the complex fugacity $\tilde{z}$ with roots which appear in complex conjugated pairs. At the transition temperature $T_{c}=1 / \beta_{c}$ these roots close in on the real axis and the density of roots determines the order of the phase transition. For the Ising model in a magnetic field $B$, Lee and Yang showed that the Lee-Yang zeros $\tilde{z}_{j}$ of the 
partition function for complex fugacity $\tilde{z}=\exp (-2 B / T)$ lie on the unit circle, $\left|\tilde{z}_{j}\right|=1$ 1314 One of the corollaries of this circle theorem is that phase transitions at a fixed temperature $T$ can only occur at zero magnetic field. A convenient way to determine the positions of the Lee-Yang zeros in the complex plane is to use a transfer matrix formulation. In this framework, the occurrence of zeros in the partition function is equivalent to a matching condition for the modulus of the two largest eigenvalues of the transfer matrix, $\left|\Lambda_{0}(\tilde{z})\right|=\left|\Lambda_{1}(\tilde{z})\right|[15 \mid 16$

In 1965, Fisher suggested to study alternatively the zeros of the canonical partition function in the complex inverse temperature plane ${ }^{[17}$ He showed that for the two-dimensional Ising model without magnetic field, the Fisher zeros also lie on a unit circle. Later, it was however realized that this is the exception rather than the rule and that no statement equivalent to the Yang-Lee circle theorem can be made ${ }^{18}$ In general, the Fisher zeros can, in addition to forming smooth curves, also densely occupy entire regions of the complex plane. The fundamental difference to the Lee-Yang study of zeros in the complex fugacity plane is that the canonical partition function is not a simple polynomial in the complex parameter $\beta$.

Many of these arguments can be transferred straightforwardly to a study of the boundary partition function, Eq. (I.3). The Fisher zeros $z_{j}$ will again determine the points where the potential

$$
f(z)=-\lim _{N \rightarrow \infty} \frac{1}{N} \ln Z(z)
$$

shows nonanalyticities. If such zeros close in on the imaginary axis, $z_{j} \rightarrow \mathrm{i} t_{j}$, nonanalyticities will also occur in the real time evolution of the quantum system and might be understood as indicating a dynamical phase transition 11 However, it is important to note that $Z(\mathrm{i} t)$, contrary to $Z_{\mathrm{th}}$, is not a strictly positive function so that $Z(\mathrm{i} t)=0$ is possible even for a finite system. An interpretation as a dynamical phase transition has been supported by analytic results for the one-dimensional transverse Ising model showing that the Fisher zeros form lines in the complex plane which cross the imaginary axis $z=\mathrm{it}$ only if the quench leads across the equilibrium quantum critical point!11 Similarly, numerical calculations for onedimensional generalized Ising models ${ }^{19}$ seem to suggest that the return rate

$$
l(t)=f(\mathrm{i} t)+f(-\mathrm{i} t)=-\lim _{N \rightarrow \infty} \frac{1}{N} \ln L(t)
$$

has nonanalyticities only for quenches across a quantum critical point. As in the two-dimensional Ising case studied by Fisher one might, however, wonder how general these results are given that the boundary partition function $Z(z)$ is not a simple polynomial in $z$.

Even for integrable models that cannot be mapped onto free fermions such as the transverse Ising model, it might be still possible to investigate the Loschmidt echo analytically in certain cases by considering the transfer matrix for the boundary partition function $Z(z)$. Indeed, it has been recently shown that an analytic result for the Loschmidt echo at imaginary times can be obtained for specific quenches in the XXZ model by applying the Bethe ansatz to the boundary transfer matrix 20 However, an analytic continuation has so far only been possible for small times. Furthermore, such an approach seems to be restricted to initial states which are products of local two-site states.

Another recent approach is based on rewriting the Loschmidt echo as a trace over a density matrix. ${ }^{21}$ For a finite system, we can write down a spectral representation of the Loschmidt amplitude

$$
Z(z)=\sum_{n}\left|\left\langle\Psi_{0} \mid n\right\rangle\right|^{2} e^{-z E_{n}}=\operatorname{Tr}\left\{\rho_{\mathrm{diag}} e^{-z H}\right\},
$$

where $\{|n\rangle\}$ are the eigenstates of $H, E_{n}$ the corresponding eigenenergies, and the diagonal ensemble is defined as

$$
\rho_{\text {diag }}=\sum_{n}\left|\left\langle\Psi_{0} \mid n\right\rangle\right|^{2}|n\rangle\langle n|
$$

From the spectral representation (I.6) one can easily obtain the long-time average of the Loschmidt echo for finite systems

$$
\bar{L} \equiv \lim _{s \rightarrow \infty} \frac{1}{s} \int_{0}^{s} L(t) d t=\sum_{n}\left|\left\langle\Psi_{0} \mid n\right\rangle\right|^{4} .
$$

Note that this is not simply the fidelity between the initial state and the ground state of the final Hamiltonian, but rather involves a sum over all eigenstates of the final Hamiltonian. In Ref 21, the diagonal ensemble in (I.6) was replaced by the generalized Gibbs ensemble $\rho_{\mathrm{GGE}}$ which is a function of all the local conservation laws. ${ }^{22}$ For a nonintegrable system the generalized Gibbs ensemble reduces to the canonical or grand canonical ensemble. ${ }^{23}$ While it was shown in Ref.21 that the Loschmidt echo for the transverse Ising model remains the same up to a factor 2 if this replacement is used, such a relation will not hold in general. The 'Loschmidt echo' with $\rho_{\text {diag }} \rightarrow \rho_{\text {GGE }}$ is a different quantity which we will not consider here.

Our paper is organized as follows: In Sec. II we describe the similarities and differences between the thermal partition function and the Loschmidt amplitude on the level of transfer matrices. Based on this representation we formulate a numerical algorithm which allows to calculate $Z(z)$ directly in the thermodynamic limit. Furthermore, we have access to the spectrum of the transfer matrix and show that nonanalyticities in $l(t)$ are a consequence of a crossing of the leading with the next leading eigenvalue. In Sec. III we give the Hamiltonian of the one-dimensional XXZ model in a uniform and staggered field which is the model we consider in the rest of the paper. This model is integrable if the staggered field is zero and nonintegrable otherwise. We recapitulate the phase diagram that includes first and second orders, as well as Berezinksy-Kosterlitz-Thouless transitions. For specific 
quenches to the free fermion point, the Fisher zeros and the Loschmidt echo can be calculated analytically. We use the analytic solution in these cases to discuss the structure of the Fisher zeros and its dependence on the initial state. Furthermore, we use bosonization to obtain analytical results for quenches within the Luttinger liquid phase and discuss the applicability of this approximation by comparing with numerical data. The results of both analytical approaches are presented in Sec.[IV] In all other cases we rely solely on numerical calculations using the transfer matrix approach introduced in Sec. II. We present, in particular, a comparison between quenches across different quantum critical lines in the XXZ model in Sec. $\mathrm{V}$ and give examples for nonanalytic behavior in $l(t)$ for quenches which stay in the same phase. The general conclusions which can be drawn from our analysis are discussed in the final section.

\section{THE TRANSFER MATRIX APPROACH}

We will first briefly revisit the transfer matrix approach to calculate the thermodynamics of one-dimensional quantum systems discussing, in particular, the crossing of eigenvalues in the spectrum of the transfer matrix. In a second step, we will extend this approach to the boundary partition function $Z(z)$.

\section{A. The finite temperature formalism}

A one-dimensional quantum model at finite temperatures can be mapped onto a two-dimensional classical model with the second dimension being given by the inverse temperature $\beta$ (imaginary time). In practice, such a mapping is achieved by a Trotter-Suzuki decomposition of the partition function 2426

$$
Z_{\mathrm{th}}=\operatorname{Tr} \mathrm{e}^{-\beta H}=\lim _{M \rightarrow \infty} \operatorname{Tr}\left(\mathrm{e}^{-\delta_{\beta} H}\right)^{M}
$$

with $\beta=\delta_{\beta} \cdot M$ fixed. The infinitesimal evolution in imaginary time can now be treated as a classical object and for a Hamiltonian with nearest-neighbor interactions, $H=\sum_{j} h_{j, j+1}$, the partition function becomes

$$
Z_{\mathrm{th}}=\lim _{M \rightarrow \infty} \operatorname{Tr}_{L}(\underbrace{\tau_{1,2}^{1,2} \tau_{2,3}^{2,3} \cdots \tau_{1,2}^{M-1, M} \tau_{2,3}^{M, 1}}_{T_{\mathrm{th}}})^{L / 2}
$$

where $L$ is the system size and we have assumed periodic boundary conditions in the spatial direction (trace " $\operatorname{Tr}_{L}$ "). In the imaginary time direction we have $M$ time slices and also periodic boundary conditions. The geometry of the classical system is therefore that of a torus as shown in Fig. 11(a).

The quantum transfer matrix $T_{\mathrm{th}}$ is two columns wide and evolves along the spatial direction. It consists of Boltzmann weights


FIG. 1: (Color online) (a) Torus geometry obtained after mapping a one-dimensional quantum system at finite temperatures with periodic boundary conditions to a twodimensional classical model. (b) Cylinder geometry required to calculate the Loschmidt amplitude. The upper and lower ends of the cylinder are fixed by the initial state $\left|\Psi_{0}\right\rangle$.

$$
\tau_{i, j}^{k, l}=\left[\exp \left(-\delta_{\beta} h_{i, j}\right)\right]_{k}^{l}
$$

with $k, l \in\{1, \cdots, M\}$ indices labeling the sites of the two-dimensional model in imaginary time direction, and $i, j \in\{1, \cdots, L\}$ indices labeling the sites in the spatial direction. We can now calculate the trace in the left and right eigenbasis $\left\{\left\langle\phi_{n}^{L}\right|\right\}$ and $\left\{\left|\phi_{n}^{R}\right\rangle\right\}$ of the $(M \times M)$ transfer matrix $T_{\text {th }}$ and find

$$
Z_{\mathrm{th}}=\lim _{M \rightarrow \infty}\left[\Lambda_{0}^{L / 2} \sum_{n=0}^{M-1}\left(\frac{\Lambda_{n}}{\Lambda_{0}}\right)^{L / 2}\right],
$$

where $\left|\Lambda_{0}\right| \geq\left|\Lambda_{1}\right| \geq \cdots \geq\left|\Lambda_{M-1}\right|$ are the eigenvalues of $T_{\mathrm{th}}$ ordered in magnitude and we have assumed that $L$ is even. For the following discussion, it is crucial to note that the eigenvalues $\Lambda_{i}(\beta)$ will always be continuous functions of inverse temperature $\beta$ if we follow their evolution without reordering them according to their modulus at every imaginary time step. For any finite system size $L$ the partition function $Z_{\mathrm{th}}$, as defined in (II.4), will therefore be an analytic function. However, in the thermodynamic limit, $L \rightarrow \infty$, the partition function will be completely determined by the largest eigenvalue $\Lambda_{0}$ giving rise to a free energy

$$
f_{\mathrm{th}}=-\lim _{L \rightarrow \infty} \frac{T}{L} \ln Z_{\mathrm{th}}=-\frac{T}{2} \ln \Lambda_{0} .
$$

Since eigenvalues can cross, this opens up the possibility for nonanalyticities. To understand the physical meaning of such crossings in the spectrum of the transfer matrix, one has to consider two-point correlation functions between two operators $A$ and $B$ given at distances $r \geq 2$ by

$$
\begin{aligned}
& \left\langle A_{1} B_{r+1}\right\rangle=\operatorname{Tr}\left\{A_{1} B_{r+1} \mathrm{e}^{-\beta H}\right\} / Z_{\mathrm{th}} \\
= & \operatorname{Tr}\left\{T_{\mathrm{th}}\left(A_{1}\right) T_{\mathrm{th}}^{[r / 2]-1} T_{\mathrm{th}}\left(B_{r+1}\right) T_{\mathrm{th}}^{L / 2-[r / 2]-1}\right\} / \operatorname{Tr} T_{\mathrm{th}}^{L / 2} \\
\stackrel{L \rightarrow \infty}{\rightarrow} & \left\langle\phi_{0}^{L}\left|T_{\mathrm{th}}\left(A_{1}\right) T_{\mathrm{th}}^{[r / 2]-1} T_{\mathrm{th}}\left(B_{r+1}\right)\right| \phi_{0}^{R}\right\rangle / \Lambda_{0}^{[r / 2]+1} \\
= & \sum_{n} \frac{\left\langle\phi_{0}^{L}\left|T_{\mathrm{th}}\left(A_{1}\right)\right| \phi_{n}^{R}\right\rangle\left\langle\phi_{n}^{L}\left|T_{\mathrm{th}}\left(B_{r+1}\right)\right| \phi_{0}^{R}\right\rangle}{\Lambda_{0} \Lambda_{n}}\left(\frac{\Lambda_{n}}{\Lambda_{0}}\right)^{[r / 2]},
\end{aligned}
$$

where $[r / 2]$ denotes the largest integer smaller than $r / 2$. $T_{\mathrm{th}}(A)$ and $T_{\mathrm{th}}(B)$ are transfer matrices as defined in 
II.2 with the operator $A$ or $B$ inserted at the appropriate position. The correlation function therefore has the asymptotic expansion

$$
\begin{aligned}
\left\langle A_{1} B_{r+1}\right\rangle & =\frac{\left\langle\phi_{0}^{L}\left|T_{\mathrm{th}}\left(A_{1}\right)\right| \phi_{0}^{R}\right\rangle\left\langle\phi_{0}^{L}\left|T_{\mathrm{th}}\left(B_{r+1}\right)\right| \phi_{0}^{R}\right\rangle}{\Lambda_{0}^{2}} \\
& +\sum_{n \geq 1} M_{n} \mathrm{e}^{i k_{n} r} \mathrm{e}^{-r / \xi_{n}}
\end{aligned}
$$

with matrix elements

$$
M_{n}=\frac{\left\langle\phi_{0}^{L}\left|T_{\mathrm{th}}\left(A_{1}\right)\right| \phi_{n}^{R}\right\rangle\left\langle\phi_{n}^{L}\left|T_{\mathrm{th}}\left(B_{r+1}\right)\right| \phi_{0}^{R}\right\rangle}{\Lambda_{0} \Lambda_{n}}
$$

wave vectors

$$
k_{n}=\frac{1}{2} \arg \left(\frac{\Lambda_{n}}{\Lambda_{0}}\right)+l \pi \quad(l=0 \text { or } l=1),
$$

and correlation lengths

$$
\xi_{n}^{-1}=\frac{1}{2} \ln \left|\frac{\Lambda_{0}}{\Lambda_{n}}\right| .
$$

A crossing of a next-leading eigenvalue $\Lambda_{n}$ with the leading eigenvalue $\Lambda_{0}$ thus implies a diverging correlation length $\xi_{n}$. For one-dimensional quantum systems there are no finite temperature phase transitions so that this will never happen and a gap between the leading and next-leading eigenvalue will persist for any finite temperature. A divergence of correlation lengths will only occur in the limit $T \rightarrow 0$ when approaching a quantum critical point or line. However, crossovers in the spectrum between eigenvalues $\Lambda_{i}$ and $\Lambda_{j}$ with $i, j>0$ are possible and lead to nonanalyticities in the correlation lengths. Such nonanalyticities have been analyzed in detail using the quantum matrix approach and Bethe ansatz for the integrable XXZ model ${ }^{27}$ and by numerical density-matrix renormalization group methods for transfer matrices (TMRG) ${ }^{28] 30}$ for the nonintegrable $t-J$ chain,,$\sqrt[3132]{32}$ spin-orbital models,,$\sqrt[33]{35}$ and an extended Hubbard model! 36

\section{B. The Loschmidt echo}

The finite temperature formalism described above can be straightforwardly generalized to a calculation of the Loschmidt amplitude $Z(z)$ defined in Eq. (I.3). There are only two modifications: (1) Instead of performing a Trotter-Suzuki decomposition for the imaginary time evolution operator $\mathrm{e}^{-\beta H}$ we now have to do such a decomposition for the evolution operator $\mathrm{e}^{-z H}$ with an arbitrary complex number $z$. (2) The geometry is different. Instead of a torus we now have to consider a cylinder with the boundaries in the $z$-direction fixed by the initial state $\left|\Psi_{0}\right\rangle$ as shown in Fig. 1(b).

In complete analogy to Eq. (II.2) we obtain

$$
Z(z)=\lim _{M \rightarrow \infty} \operatorname{Tr}_{L} \bar{T}^{L / 2}
$$

where the trace is taken along the spatial direction due to the assumed periodic boundary conditions. The quantum transfer matrix $\bar{T}$ now has boundary degrees of freedom fixed by the initial configuration $\left|\Psi_{0}\right\rangle$,

$$
\bar{T}=\widetilde{\tau}_{1,2}^{1,2} \bar{\tau}_{2,3}^{2,3} \cdots \bar{\tau}_{1,2}^{M-1, M} \widetilde{\tau}_{2,3}^{M, M+1}
$$

with weights $\bar{\tau}_{i, j}^{k, l}$ as given in Eq. II.3 with $\delta_{\beta} \rightarrow \delta_{z}$. The first and last weight $\widetilde{\tau}$ are, however, modified with matrixelements

$$
\widetilde{\tau}_{1,2}^{1,2}=\left\langle s_{1}^{1} s_{2}^{1}\left|\exp \left(-\delta_{z} h_{1,2}\right)\right| s_{1}^{2} s_{2}^{2}\right\rangle
$$

where the degrees of freedom $s_{1}^{1}$ and $s_{2}^{1}$ are fixed by the initial state $\left|\Psi_{0}\right\rangle . \widetilde{\tau}_{2,3}^{M, M+1}$ is defined analogously.

For the boundary partition function $Z(z)$ the formula (II.4) thus applies as well with the eigenvalues $\Lambda_{i}$ of the thermal transfer matrix $T_{\text {th }}$ replaced by the eigenvalues $\bar{\Lambda}_{i}$ of the boundary transfer matrix $\bar{T}$. If we order these eigenvalues again by magnitude, $\left|\bar{\Lambda}_{0}\right| \geq\left|\Lambda_{1}\right| \geq \ldots \geq$ $\left|\bar{\Lambda}_{M-1}\right|$, then the return rate $f(z)$ defined in Eq. (I.4) is given in the thermodynamic limit by

$$
f(z)=-\frac{1}{2} \lim _{M \rightarrow \infty} \ln \bar{\Lambda}_{0}
$$

However, there is now no longer necessarily a gap between the largest and next-leading eigenvalues. Crossings are possible which can make $\bar{\Lambda}_{0}(z)$ and thus $f(z)$ a nonanalytic function. In order to find the lines where the Fisher zeros accumulate in the thermodynamic limit we therefore have to find the $z_{0}$ values for which

$$
\left|\bar{\Lambda}_{0}\left(z_{0}\right)\right|=\left|\bar{\Lambda}_{k}\left(z_{0}\right)\right| \text { and }\left|\bar{\Lambda}_{0}\left(z_{0} \pm \delta\right)\right| \neq\left|\bar{\Lambda}_{k}\left(z_{0} \pm \delta\right)\right|
$$

with $|\delta| \ll 1$. In this case, we have two regions where either $\bar{\Lambda}_{0}$ or $\bar{\Lambda}_{k}$ dominate with the matching condition (II.15) leading to a nonanalyticity in $f(z)$. Note that in both regions the largest eigenvalue can be degenerate. Only a true crossing - not a degeneracy - leads to nonanalytic behavior. Alternatively, we can understand the crossing of two eigenvalues as a divergence of a correlation length

$$
\bar{\xi}_{k}^{-1}=\frac{1}{2} \ln \left|\frac{\bar{\Lambda}_{0}}{\bar{\Lambda}_{k}}\right|
$$

In this sense, the crossing is indeed indicating a dynamical phase transition. Finally, we want to explicitly give the formula for the return amplitude in the thermodynamic limit,

$$
l(t)=f(\mathrm{i} t)+f(-\mathrm{i} t)=-\frac{1}{2} \ln \left|\bar{\Lambda}_{0}\right|^{2} .
$$

In the next section we describe a numerical algorithm to calculate the boundary transfer matrix $\bar{T}$. Using Eqs. II.15 and (II.17) we can then determine numerically the Fisher zeros and the return amplitude for any translationally invariant one-dimensional model with short-range interactions directly in the thermodynamic limit. 


\section{The numerical implementation}

For the numerical simulations, we use the light cone renormalization group (LCRG) algorithm introduced in Ref 37. Working in the thermodynamic limit, we have direct access to the transfer matrix $\bar{T}$, from which we can calculate the largest eigenvalues $\bar{\Lambda}_{n}$ using an iterative Lanczos eigensolver. The LCRG algorithm was originally introduced to calculate expectation values of observables $O$ following a quantum quench, $\langle O(t)\rangle=$ $\left\langle\Psi_{0}\left|\mathrm{e}^{i H t} O \mathrm{e}^{-i H t}\right| \Psi_{0}\right\rangle$. In this case the corresponding twodimensional classical model has the form of a light cone with Boltzmann weights of the forward and backward time evolution canceling outside of the light cone which has the operator $O$ at its center. No such structure exists for the Loschmidt amplitude where only the evolution operator forward in time is present. The transfer matrix in the LCRG algorithm, which evolves along the horizon of the light cone, can nevertheless still be used to construct a two-dimensional lattice which is infinite in the spatial direction and the relation (II.14) remains valid. Alternatively, one can use an infinite time evolving block decimation (iTEBD) algorithm ${ }^{38}$ to construct the transfer matrix $\bar{T}$. This method has been used to calculate $l(t)$ in the thermodynamic limit for generalized Ising models in Ref 19. However, in this work only the real-time evolution has been considered while we will use a general complex parameter $z$ in the evolution operator which gives us, in addition, also access to the Fisher zeros in the complex plane.

The ground state of the initial Hamiltonian is obtained in our algorithm by applying an imaginary time evolution $e^{-\beta H}$ to an arbitrary state. For gapped initial Hamiltonians, we have $e^{-\beta H}=\left|\Psi_{0}\right\rangle\left\langle\Psi_{0}\right|+\mathcal{O}\left(e^{-\beta \Delta}\right)$, with $\Delta=E_{1}-E_{0}$ the energy gap. In this case the convergence is fast once imaginary times $\beta \gg 1 / \Delta$ are reached. For an initial state in a gapless phase, this projection method is much more problematic and can be the source of significant numerical errors ${ }^{39}$ In the integrable case, we will check the convergence by comparing with the exact ground-state energy ${ }^{40}$ The projection onto the ground state is followed by the standard time evolution with $U(z)=e^{-z H}$, where $z=R+$ it is a complex parameter. All simulations are carried out using an adaptive block dimension of up to $\chi=768$ states such that the discarded weight at each step remains smaller than $10^{-15}$. We can, in addition, get an upper bound on the possible simulation time $t_{\max }$ by calculating the entanglement entropy $S_{\text {ent }}$. For a block dimension $\chi$, the maximal entanglement entropy which can be represented is given by $S_{\mathrm{ent}}^{\max }=\ln \chi$. Since $S_{\mathrm{ent}}(t)=c t$ with a constant $c$, we can easily determine $t_{\max }$ by the condition $c t_{\max }=S_{\text {ent }}^{\max }$ and we make sure that in our simulations $t$ is always much smaller than $t_{\max }$.

To show that it is indeed possible to determine the lines of Fisher zeros using the condition II.15 and the LCRG algorithm, we consider the one-dimensional transverse Ising model

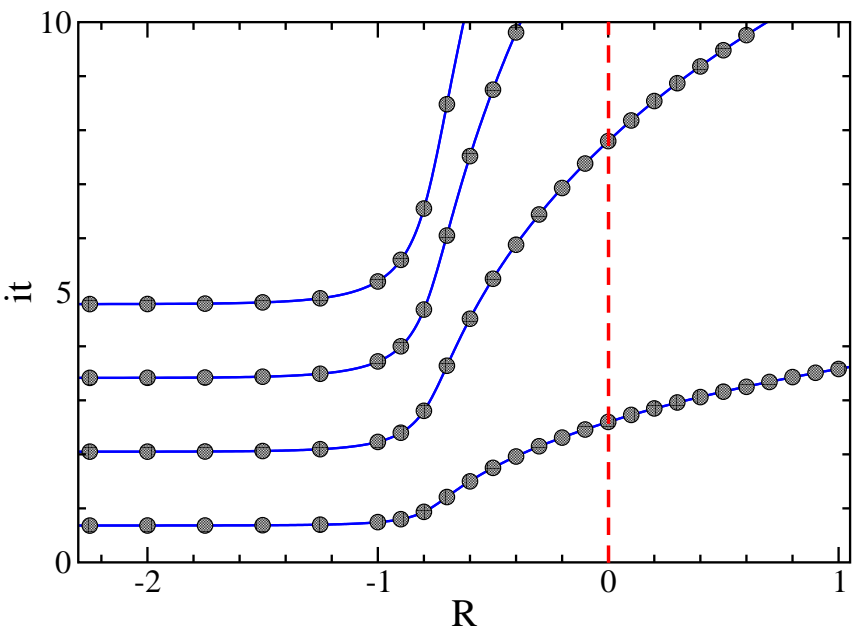

FIG. 2: Lines of Fisher zeros in the complex plane, $z=R+i t$, for a quench $g=0.4 \rightarrow 1.3$ in the transverse Ising model (II.18). The exact results 11 (lines) are compared with numerical scans through the complex plane using the LCRG algorithm (circles).

$$
H=-\frac{1}{2} \sum_{i} \sigma_{i}^{z} \sigma_{i+1}^{z}+\frac{g}{2} \sum_{i} \sigma_{i}^{x}
$$

where an analytical solution can be obtained!1011 In Fig. 2, the analytical result taken from Ref 11 is compared with numerical data obtained by the LCRG algorithm for a quench across the quantum phase transition at $g=1$, showing excellent agreement.

\section{THE MODEL}

In order to exemplarily investigate the Fisher zeros, the nonanalyticities in the Loschmidt echo, and their relation to equilibrium quantum phase transitions, we consider quenches in a spin-1/2 XXZ model given by

$$
\begin{aligned}
H & =J \sum_{j}\left\{\frac{1}{2}\left[S_{j}^{+} S_{j+1}^{-}+S_{j+1}^{+} S_{j}^{-}\right]+\Delta S_{j}^{z} S_{j+1}^{z}\right\} \\
& +\sum_{j}\left(h+(-1)^{j} h_{\mathrm{st}}\right) S_{j}^{z} .
\end{aligned}
$$

Here $J$ is the exchange coupling constant, $\Delta$ parametrizes the exchange anisotropy, $h$ is a uniform, and $h_{\text {st }}$ is a staggered magnetic field. We will concentrate on investigating the model for the following two cases: (I) $h_{\mathrm{st}}=0$. In this case, the model is integrable by Bethe ansatz. The phase diagram, shown schematically in Fig. 3(a), consists of a ferromagnetic (FM), a Luttinger liquid (LL), and an antiferromagnetic (AFM) phase 40 The phase transitions are of first, second, and Berezinsky-Kosterlitz-Thouless (BKT) type as indicated in Fig. 3(a). (II) $h=0$ and $h_{\mathrm{st}} \neq 0$. In this case, the model is not integrable. Again, we have FM, LL, and AFM phases, separated by first- 



FIG. 3: Schematic phase diagrams for the Hamiltonian (III.1) for (a) the integrable case $h_{\mathrm{st}}=0$, and (b) the nonintegrable case with staggered field but $h=0$. The phase diagrams consist of gapped ferromagnetic (FM) and antiferromagnetic (AFM) phases as well as a gapless Luttinger liquid (LL) phase. In (a), the transition at $\Delta=1$ and $h=0$ is of BerezinskyKosterlitz-Thouless (BKT) type, while in (b), the whole line between AFM and LL phase is a BKT transition.

order, second-order, and BKT transitions as indicated in the schematic phase diagram in Fig. 3 (b) $\underline{41 / 42}$

It is clear right from the start that the Loschmidt echo is trivial for certain quenches across those phase transitions. First, this is always the case for quenches starting in the FM phase because the ferromagnetic state is always an eigenstate of the Hamiltonian (III.1). No matter what parameters we choose for the final Hamiltonian, we will always obtain $|Z(z)| \equiv 1$. Furthermore, $|Z(z)|$ is also always equal to one if we only quench the uniform magnetic field, $h_{\text {ini }} \rightarrow h_{\text {fin }}$, because $\left[H, \sum_{j} S_{j}^{z}\right]=0$. There is thus no direct correspondence between the zero temperature phase transitions and nonanalyticities in the Loschmidt echo in general. In Sec. $\mathrm{V}]$ we will even show that nonanalyticities in the Loschmidt echo can occur for this model for quenches which do not cross any of the transition lines. In the following, we will denote the quench from a given set of initial to some final microscopic parameters by $\left(\Delta_{\text {ini }}, h_{\text {ini }}, h_{\text {st,ini }}\right) \rightarrow\left(\Delta_{\text {fin }}, h_{\text {fin }}, h_{\text {st }, \text { fin }}\right)$ and set $J=1$.

\section{ANALYTICAL RESULTS FOR BILINEAR HAMILTONIANS}

Before numerically investigating the model (III.1), first we want to discuss cases where we can exactly calculate $Z(z)$ or where we might be able to approximate the exact time evolution by that of a bilinear Hamiltonian obtained by bosonization.

\section{A. Free fermion case}

For $\Delta=0$, we can map the Hamiltonian (III.1) to a free fermion Hamiltonian using the Jordan-Wigner transformation

$$
S_{j}^{+}=(-1)^{j} c_{j}^{\dagger} \mathrm{e}^{i \pi \phi_{j}}, S^{-}=(-1)^{j} c_{j} \mathrm{e}^{-i \pi \phi_{j}}, S_{j}^{z}=n_{j}-1 / 2
$$

with $n_{j}=c_{j}^{\dagger} c_{j}=S_{j}^{+} S_{j}^{-}, \phi_{j}=\sum_{l=1}^{j-1} n_{l}$, where $c_{j}\left(c_{j}^{\dagger}\right)$ are annihilation (creation) operators of spinless fermions at site $j$. In the following we assume that the spin chain has an even number of lattice sites $N$ and periodic boundary conditions. Equation (IV.1) then leads to the following fermionic Hamiltonian

$$
\begin{aligned}
H_{0} & =-\frac{J}{2}\left[\sum_{j=1}^{N-1}\left(c_{j}^{\dagger} c_{j+1}+h . c .\right)-\mathrm{e}^{i \pi M}\left(c_{N}^{\dagger} c_{1}+h . c .\right)\right] \\
& +\sum_{j=1}^{N}\left[h+(-1)^{j} h_{\mathrm{st}}\right]\left(n_{j}-1 / 2\right)
\end{aligned}
$$

where $M=\sum_{j=1}^{N} n_{j}$ is the total particle number. Note that the boundary conditions for the fermions are only periodic if $M$ is odd. Otherwise there is a phase shift $\underline{43} \mathrm{In}$ order to diagonalize $H_{0}$ we can define a Fourier transform as

$$
c_{j}=\frac{1}{\sqrt{N}} \sum_{n=1}^{N} \mathrm{e}^{-i j\left(k_{n}+a\right)}
$$

where $k_{n}=2 \pi n / N, a=\pi / N$ for $M$ even, and $a=0$ for $M$ odd, leading to

$$
\begin{aligned}
H_{0} & =\sum_{n=1}^{N}\left(\varepsilon_{k_{n}}-h\right) c_{k_{n}}^{\dagger} c_{k_{n}}-h_{\mathrm{st}} \sum_{n=1}^{N} c_{k_{n}}^{\dagger} c_{k_{n+N / 2}} \\
& =\sum_{n=1}^{N / 2}\left[\left(\varepsilon_{k_{n}}-h\right) c_{k_{n}}^{\dagger} c_{k_{n}}-\left(\varepsilon_{k_{n}}+h\right) d_{k_{n}}^{\dagger} d_{k_{n}}\right] \\
& -h_{\mathrm{st}} \sum_{n=1}^{N / 2}\left[c_{k_{n}}^{\dagger} d_{k_{n}}+d_{k_{n}}^{\dagger} c_{k_{n}}\right]
\end{aligned}
$$

with $\varepsilon_{k_{n}}=-J \cos \left(k_{n}+a\right)$. In the second and third lines, we have introduced new operators $d_{k_{n}}$ by folding the band back into a reduced Brillouin zone. This bilinear Hamiltonian can now easily be diagonalized by a rotation

$$
\begin{aligned}
H_{0} & =\sum_{n=1}^{N / 2}\left[\left(\sqrt{\varepsilon_{k_{n}}^{2}+h_{\mathrm{st}}^{2}}-h\right) \alpha_{k_{n}}^{\dagger} \alpha_{k_{n}}\right. \\
& \left.-\left(\sqrt{\varepsilon_{k_{n}}^{2}+h_{\mathrm{st}}^{2}}+h\right) \beta_{k_{n}}^{\dagger} \beta_{k_{n}}\right]
\end{aligned}
$$

Note that the rotation needed to diagonalize the Hamiltonian is a function of the microscopic parameters $J, h, h_{\mathrm{st}}$.

Now we can consider various quenches in the free fermion model (IV.5) by changing these parameters. We start with a particularly simple case where the initial state $\left|\Psi_{0}\right\rangle$ is the ground state of $H_{0}$ with $J=h=0$ and $h_{\text {st }} \neq 0$. This is nothing but a Néel state that we can express as $\left|\Psi_{0}\right\rangle=\prod_{n} \beta_{k_{n}}^{\dagger}|0\rangle$ using the operators defined in (IV.5). As the final Hamiltonian we take the Hamiltonian in IV.4 for $h=h_{\mathrm{st}}=0$, which is already diagonal. In this case the relation between the old and new operators is particularly simple: 




FIG. 4: (Color online) The return rate $l(t)$, Eq. (IV.8), for a system with $N=120$ sites. $l(t)$ diverges at times $t_{c}$ given by Eq. IV.9.

$$
c_{k_{n}}=\frac{1}{\sqrt{2}}\left(\alpha_{k_{n}}+\beta_{k_{n}}\right), \quad d_{k_{n}}=\frac{1}{\sqrt{2}}\left(\alpha_{k_{n}}-\beta_{k_{n}}\right) .
$$

The time evolution of the initial state is now trivially given by

$$
\mathrm{e}^{-z H}\left|\Psi_{0}\right\rangle=\frac{1}{\sqrt{2}} \prod_{n=1}^{N / 2}\left[\left(\mathrm{e}^{-z \varepsilon_{k_{n}}} c_{k_{n}}^{\dagger}-\mathrm{e}^{z \varepsilon_{k_{n}}} d_{k_{n}}^{\dagger}\right)\right]|0\rangle
$$

and the Loschmidt amplitude becomes $Z(z)=$ $\prod_{n} \cosh \left(z \varepsilon_{k_{n}}\right)$. For the return rate this yields

$$
\begin{aligned}
& l(t)=-\frac{1}{N} \sum_{n=1}^{N / 2} \ln \cos ^{2}\left(t \varepsilon_{k_{n}}\right) \\
& \stackrel{N \rightarrow \infty}{\rightarrow}-\frac{1}{2 \pi} \int_{-\pi / 2}^{\pi / 2} d k \ln \cos ^{2}\left(t \varepsilon_{k}\right) .
\end{aligned}
$$

Here we want to emphasize again an important difference to the thermal partition function. While $Z_{\mathrm{th}}(z)$ can never have zeros on the real axis, the Loschmidt amplitude $Z(z)$ can become zero for $z=\mathrm{i}$. This simply means that the time evolved state has become orthogonal to the initial state. From (IV.8), we see that this indeed happens for the considered quench and finite system size $N$ at times

$$
t_{c}=\frac{\pi}{2 \varepsilon_{k_{n}}}(2 m+1), \quad m \in \mathbb{Z}, n=0, \cdots, N-1 .
$$

At these times, the return rate $l(t)$ diverges, see Fig. 4. In the thermodynamic limit, however, only cusps in the return rate $l(t)$ remain. The integral in (IV.8) is dominated by contributions from the band edge, $k \approx 0$, and cusps occur if $\cos (t \cos (k=0))=0$, i.e., at times

$$
t_{c}=\frac{\pi}{2}(2 m+1)
$$

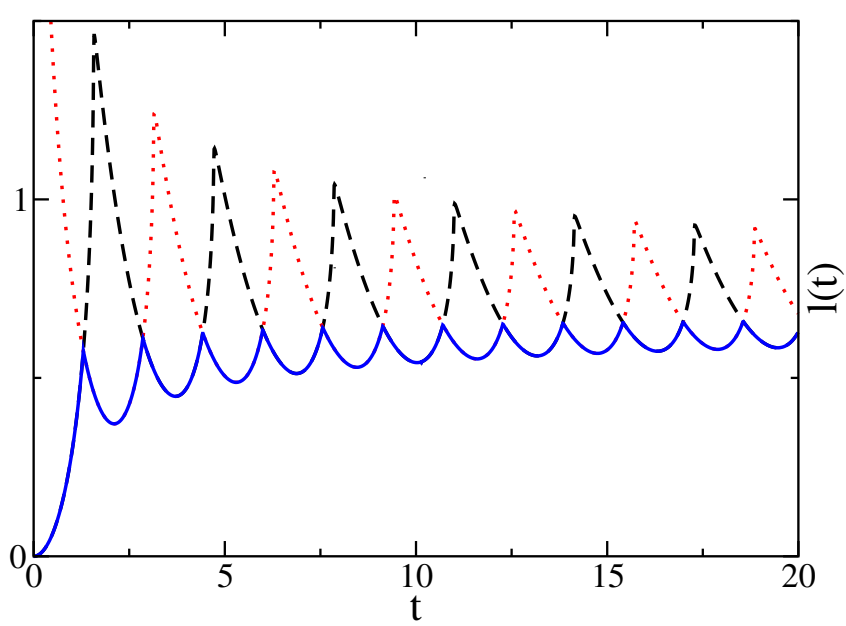

FIG. 5: (Color online) The return rate $l(t)$ in the thermodynamic limit, Eq. IV.8) (dashed line), the two contributions contributions in Eq. (IV.13) shown separately (dashed and dotted lines), and the minimum of these two contributions (solid line).

as shown in Fig. 5 (dashed line).

The location of the Fisher zeros depends, however, very sensitively on the initial state. For the Néel state chosen above the zeros all lie on the real time axis. This changes completely if we consider the symmetric combination of the two possible Néel states, i.e.,

$$
\left|\Psi_{0}\right\rangle=\frac{1}{\sqrt{2}}\left(\prod_{n} \beta_{k_{n}}^{\dagger}+\prod_{n} \alpha_{k_{n}}^{\dagger}\right)|0\rangle .
$$

In this case the Loschmidt amplitude becomes

$$
Z(z)=\prod_{n} \cosh \left(z \varepsilon_{k_{n}}\right)+\prod_{n} \sinh \left(z \varepsilon_{k_{n}}\right) .
$$

In the thermodynamic limit, always one of the two contributions dominate depending on the parameter $z$. The return rate is, in particular, given by

$$
\begin{aligned}
l(t)=-\frac{1}{2 \pi} \min \{ & \int_{-\pi / 2}^{\pi / 2} d k \ln \cos ^{2}\left(t \varepsilon_{k}\right), \\
& \left.\int_{-\pi / 2}^{\pi / 2} d k \ln \sin ^{2}\left(t \varepsilon_{k}\right)\right\}
\end{aligned}
$$

and is shown in Fig. 5 (solid line). Fisher zeros in the complex plane occur if the two contributions in (IV.13), taken as function of the complex parameter $z=R+\mathrm{i}$, are of equal magnitude and are therefore determined by

$$
0=\int_{0}^{\pi / 2} d k \ln \left|\tanh \left(z \varepsilon_{k}\right)\right|^{2}
$$

A plot of the Fisher zeros for this quench, obtained by numerically solving (IV.14, is shown in Fig. 6. The sep- 




FIG. 6: Lines of Fisher zeros obtained by Eq. IV.14 for the quench from the symmetric Néel state (IV.11) to the free fermion point (solid lines), and Fisher zeros at times $t_{c}$, Eq. IV.10, for the quench from the polarized state (symbols).

arate Fisher zeros at real times $t_{c}$ given by Eq. (IV.10) for the quench from the Néel state thus turn into closed lines in the complex plane if we instead quench from the superposition of Néel states, Eq. (IV.11.

\section{B. Bosonization in the Luttinger liquid phase}

A one-dimensional quantum system with gapless excitations can be described at low energies as a Luttinger liquid $\underline{44}$

$$
\begin{aligned}
H_{b} & =v_{F} \sum_{q>0} q\left\{\left(1+\frac{g_{4}}{2 \pi v_{F}}\right)\left[b_{R q}^{\dagger} b_{R q}+b_{L q}^{\dagger} b_{L q}\right]\right. \\
& \left.+\frac{g_{2}}{2 \pi v_{F}}\left[b_{R q}^{\dagger} b_{L q}^{\dagger}+b_{L q} b_{R q}\right]\right\} .
\end{aligned}
$$

Here $v_{F}$ is the Fermi velocity, and the bosonic operators $b_{R / L, q}$ describe the right/left moving collective excitations with momentum $q \cdot g_{2,4}$ are forward scattering amplitudes.

We set $\bar{g}_{i}=g_{i} /\left(2 \pi v_{F}\right)$ and consider a quench where the scattering amplitudes are changed from values $\bar{g}_{i, 1}$ to $\bar{g}_{i, 2}$ in a quench. For both sets of parameters the Hamiltonian IV.15 is bilinear and can be diagonalized by a Bogoliubov transform:

$$
\alpha_{i q}^{R / L}=u_{i} b_{R / L, q}+v_{i} b_{L / R, q}^{\dagger}
$$

with parameters

$$
v_{i}^{2}=\frac{1}{2}\left[\frac{1+\bar{g}_{4, i}}{\sqrt{\left(1+\bar{g}_{4, i}\right)^{2}-\bar{g}_{2, i}^{2}}}+1\right]=\frac{\left(1+K_{i}\right)^{2}}{4 K_{i}},
$$

$u_{i}^{2}=v_{i}^{2}-1$, and $i=1,2$. In Eq. (IV.17) we have also introduced the Luttinger parameters $K_{i}$.

The two new operator sets $\alpha_{1 q}^{R / L}$ and $\alpha_{2 q}^{R / L}$ are then again related by a Bogoliubov transform,

$$
\begin{aligned}
\alpha_{1 q}^{L \dagger} & =\left(v_{1} u_{2}-u_{1} v_{2}\right) \alpha_{2 q}^{R}+\left(u_{1} u_{2}-v_{1} v_{2}\right) \alpha_{2 q}^{L \dagger} \\
\alpha_{1 q}^{R} & =(\underbrace{u_{1} v_{2}-v_{1} u_{2}}_{v^{\prime}}) \alpha_{2 q}^{L \dagger}+(\underbrace{u_{1} u_{2}-v_{1} v_{2}}_{u^{\prime}}) \alpha_{2 q}^{R} .
\end{aligned}
$$

Now it is straightforward to calculate the Loschmidt amplitude

$$
\begin{aligned}
Z(z) & ={ }_{1}\left\langle 0\left|e^{-z H_{2}}\right| 0\right\rangle_{\alpha_{1}} / \alpha_{1}\langle 0 \mid 0\rangle_{\alpha_{1}} \\
& =\alpha_{2}\left\langle 0\left|T^{\dagger} e^{-z H_{2}} T\right| 0\right\rangle_{\alpha_{2}} / \alpha_{2}\left\langle 0\left|T^{\dagger} T\right| 0\right\rangle_{\alpha_{2}}
\end{aligned}
$$

with

$$
T=\exp \left\{\frac{v^{\prime}}{c^{\prime}} \sum_{q} \alpha_{2 q}^{R \dagger} \alpha_{2 q}^{L \dagger}\right\}
$$

where $|0\rangle_{\alpha_{1}}$ is the ground state before the quench and $H_{2}$ the Hamiltonian after the quench given by

$$
H_{2}=v \sum_{q>0} q\left(\alpha_{2 q}^{R \dagger} \alpha_{2 q}^{R}+\alpha_{2 q}^{L \dagger} \alpha_{2 q}^{L}\right)
$$

with a renormalized velocity $v=v_{F} \sqrt{\left(1+\bar{g}_{4,2}\right)^{2}-\bar{g}_{2,2}^{2}}$. For the Loschmidt amplitude this leads to

$$
Z(z)=\prod_{q>0} \frac{1-\bar{K}^{2}}{1-\bar{K}^{2} \mathrm{e}^{-2 z v q}} \text { with } \bar{K}=\frac{K_{1}-K_{2}}{K_{1}+K_{2}} .
$$

We see that for a quench within the Luttinger model, $Z(z)$ does not have any zeros in the complex plane.

For the XXZ model (III.1) with $h_{\text {st }}=0$, integrability makes it possible to determine the Luttinger parameter $K$ and the velocity $v$ exactly as a function of the anisotropy $\Delta$ :

$$
v=\frac{\pi}{2} \frac{\sqrt{1-\Delta^{2}}}{\arccos \Delta}, \quad K=\frac{\pi}{2(\pi-\arccos \Delta)} .
$$

We can thus try to compare the return rate 45

$$
l_{\mathrm{Bos}}(t, \Lambda)=-\frac{1}{2 \pi} \int_{0}^{\Lambda} \ln \left\{\frac{\left(1-\bar{K}^{2}\right)^{2}}{1+\bar{K}^{4}-2 \bar{K}^{2} \cos (2 t v q)}\right\}
$$

with numerical data for quenches within the critical phase. However, it is important to stress that sudden quenches are in general not low-energy problems and we therefore cannot expect Luttinger liquid theory to work. Only for small quenches, where a small amount of energy is put into the system, might (IV.24) yield a reasonable description. This problem is evident in Eq. (IV.24) where 
we have to use some cutoff scheme to make the integral convergent at short wavelengths. The result (IV.24) is thus nonuniversal and depends on the properties of the microscopic model on the scale of the lattice constant. Here, we have simply introduced a hard cutoff $\Lambda$, which we will use as a fitting parameter when comparing with the numerical data in the next section.

\section{NUMERICAL RESULTS}

We now investigate the model (III.1) using the LCRG algorithm described in Sec. II C.

\section{A. Integrable case}

We will first concentrate on quenches where the Hamiltonian in the time evolution operator $U(t)$ is given by the integrable XXZ model, Eq. (III.1) with $h_{\text {st }}=0$. For a chain of length $N$ this model has $N$-many local conserved charges, i.e., conserved charges which can be written as a sum over a density operator with finite support. $18[49$ In addition, it has been recently shown that in the critical regime, $-1<\Delta<1$, a set of quasilocal conserved charges exist.50|51 These local and quasilocal conservation laws do affect the thermalization of a quantum system at long times after a quantum quench and have to be included in the statistical ensemble. ${ }^{23|52| 53}$ In the XXZ model, the thermal current is, for example, one of the local conserved charges. If we prepare the system in an initial state $\left|\Psi_{0}\right\rangle$ with a finite thermal current then this current will remain nonzero for all times in the time evolved state $|\Psi(t)\rangle$ while it would decay in a generic quantum system. From this example it is obvious that the Loschmidt amplitude, $\left\langle\Psi_{0} \mid \Psi(t)\right\rangle$, can behave very differently depending on whether or not the Hamiltonian in the time evolution operator is integrable. It therefore makes sense to consider the integrable and nonintegrable cases separately.

\section{Quenches within the critical phase}

We start by considering small quenches in the Luttinger liquid phase which can be compared with the prediction by bosonization. To obtain the ground state, we use the projection method described in Sec. II C. The relative error, $\Delta e_{\text {rel }}=\left|\left(e_{0}-e_{\mathrm{BA}}\right) / e_{\mathrm{BA}}\right|$, in the numerically obtained ground-state energy $e_{0}$ in comparison to the exact one, $e_{\mathrm{BA}}$, known from the Bethe ansat $2{ }^{40}$ is given in Table I.

In the long-time limit bosonization predicts that the return rate $l(t)=f(\mathrm{i} t)+f(-\mathrm{i} t)$ saturates at $\frac{47}{4}$

$$
\lim _{t \rightarrow \infty} l(t)=-\frac{\Lambda}{\pi} \ln \left(1-\bar{K}^{2}\right) .
$$

By demanding that this agrees with the numerically found saturation value, we "fix" the cutoff $\Lambda$. The val-

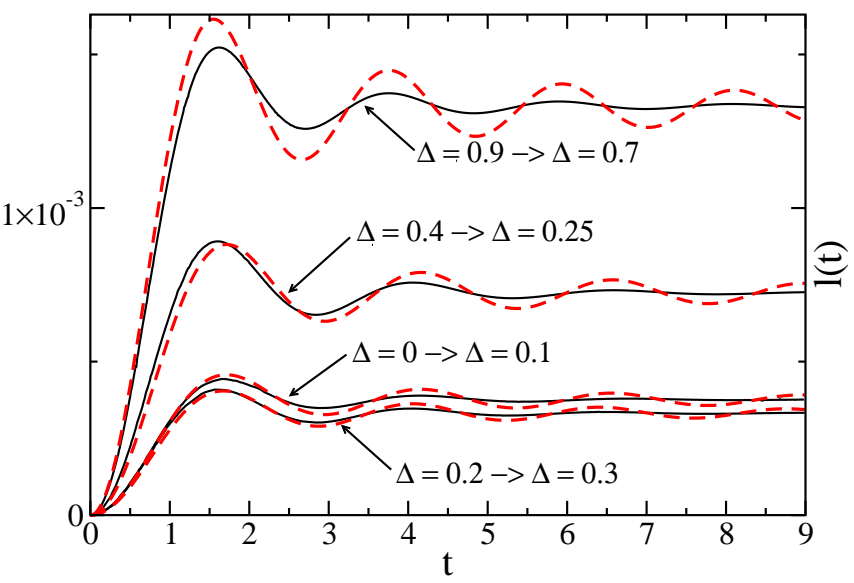

FIG. 7: (Color online) Comparison between LCRG data (solid lines) and the bosonization result, $l_{\mathrm{Bos}}(t, \Lambda)$ (dashed lines), with fit parameters $\Lambda$ and a velocity shift $v_{0}$ as given in table I.

\begin{tabular}{c|c|c|c|c}
\hline \hline$\Delta_{\text {ini }}$ & $\Delta e_{\text {rel }}$ & $\Delta_{\text {fin }}$ & $\Lambda$ & $v_{0}$ \\
\hline \hline 0 & $2.5 \cdot 10^{-3}$ & 0.1 & 1.235 & 0.0 \\
\hline 0.2 & $1.2 \cdot 10^{-3}$ & 0.3 & 1.3 & -0.15 \\
\hline 0.4 & $1.3 \cdot 10^{-3}$ & 0.25 & 1.305 & -0.15 \\
\hline 0.9 & $8.6 \cdot 10^{-3}$ & 0.7 & 0.89 & 0.22 \\
\hline \hline
\end{tabular}

TABLE I: The fit parameters $\Lambda$ and $v_{0}$ used in the comparison of the numerical data for the return rate $l(t)$ and the bosonization result (IV.24), see Fig. 7

ues that we find from this condition are also given in Table I. In addition, we allow for a shift in the oscillation frequency by replacing $v \rightarrow v+v_{0}$ in Eq. (IV.24) with $v_{0}$ used as an additional fitting parameter. Such a shift can be expected due to irrelevant operators that have not been taken into account in the Luttinger model. The results of such fits are shown in Fig. 7 and the fit parameter $v_{0}$ is given in Table[1. Note that the Luttinger parameters $K_{1}$ and $K_{2}$ as well as the velocity $v$ of the collective excitations in the final Hamiltonian are fixed by Eq. (IV.23).

While such fits are in reasonable agreement with the numerical data, we want to stress that the predictive power of the bosonization result is limited. In particular, the frequency of the oscillations in $l(t)$ depends on the long wavelength cutoff $\Lambda$. Furthermore, the numerical data seem to show that the oscillations are much stronger damped than predicted at this level by bosonization with the damping becoming more pronounced the larger the interaction $\Delta$ is.

\section{Quenches across the first-order transition}

The XXZ model with $h=h_{\text {st }}=0$ has a first-order transition between the Luttinger liquid and the ferromagnetic phase at $\Delta=-1$. For a quench starting in the 


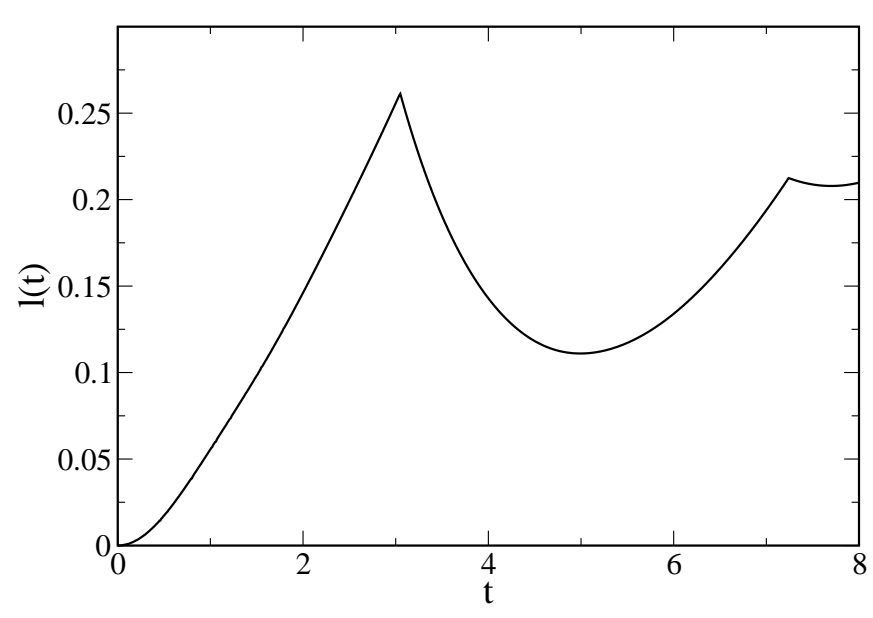

FIG. 8: (Color online) Return rate $l(t)$ for a quench from the Luttinger liquid phase, $\Delta=-0.8$, into the ferromagnetic phase, $\Delta=-2.0$.

FM phase, we have $|Z(z)|=1$ because the FM state is an eigenstate of the XXZ Hamiltonian for all interaction strengths $\Delta$. This is a trivial example showing that a phase transition does not necessarily imply the existence of Fisher zeros or nonanalyticities in the return rate.

Nontrivial results are, on the other hand, obtained for quenches from the LL into the FM phase. Numerically, the calculations for such quenches are again demanding because the projection method described in Sec. IIC to obtain the ground state converges rather slowly in a gapless phase. In Fig. 8 we show $l(t)$ for such a quench where we have obtained $\Delta e_{\text {rel }}=4 \times 10^{-4}$ for the ground-state energy of the initial state. Two cusps in $l(t)$ are clearly visible in this case indicating a dynamical phase transition as defined by the divergence of $\bar{\xi}_{k}$, see Eq. (II.16).

\section{Quenches across the BKT transition}

At $\Delta=1$, the XXZ model in zero field shows a BKT transition between the LL and the AFM phase driven by a marginal Umklapp scattering term. The excitation gap on the AFM site is opening up exponentially slowly, $\Delta E \sim \exp [-1 /(\Delta-1)]$, and derivatives of the energy do not show any singularities at finite order. If cusps in the return rate are indeed related to quantum phase transitions one might therefore wonder if they will also be present for such an infinite order transition. In Figs. 9 and 10, we present an example for a quench from the AFM into the LL phase. As an initial state, we can either take the ground state where the $S_{j}^{z} \rightarrow-S_{j}^{z}$ symmetry is broken (polarized state) or a state where this symmetry is preserved (symmetric state) while the $U(1)$ symmetry (rotations in the $x-y$ plane) always remains intact. In both cases, the leading and next-leading eigenvalues cross, leading to cusps in the return rate. The positions and the form of the cusps is, however, very different depending on whether we choose the polarized or the sym-

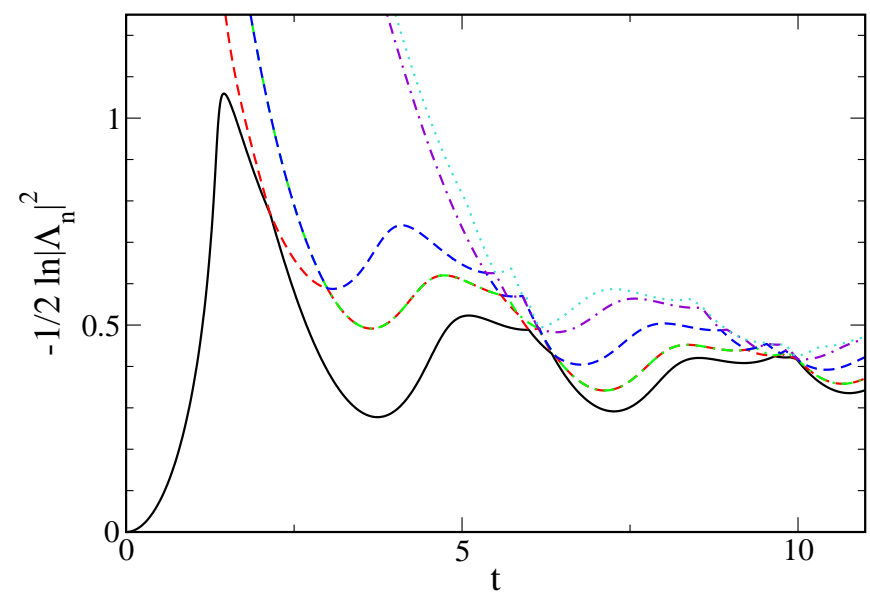

FIG. 9: (Color online) Return rate $l(t)$ and next-leading eigenvalues, $-\left(\ln \left|\Lambda_{n}\right|^{2}\right) / 2$, for a quench from the AFM to the LL phase. Here $\Delta_{\text {ini }}=6$, with the initial state being polarized, and $\Delta_{\text {fin }}=-0.6$.

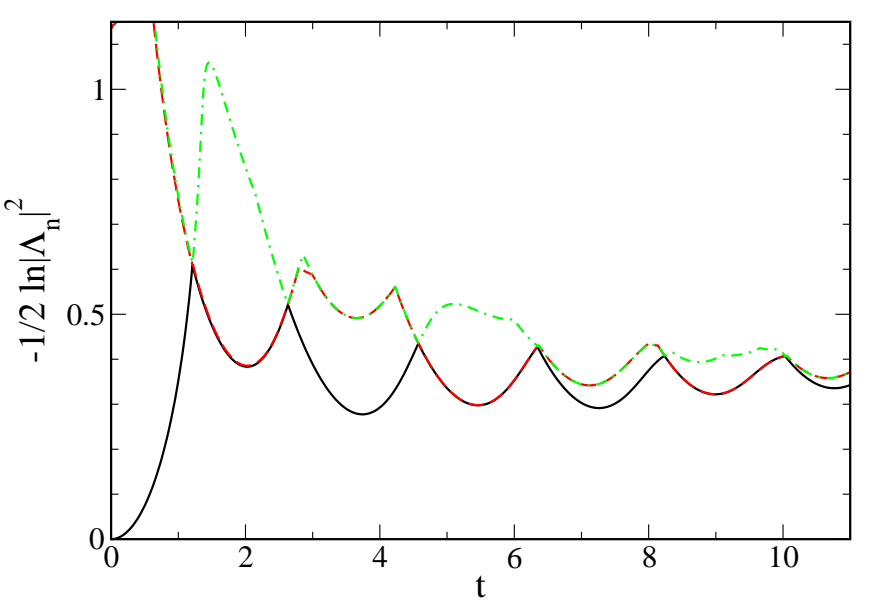

FIG. 10: (Color online) Same quench as shown in Fig. 9 but with a symmetric initial state. The eigenvalues shown are twofold degenerate.

metric initial state. A similar dependence on the symmetry of the initial ground state has also been observed previously for generalized Ising model $\frac{19}{19}$ and in the quench to the free fermion point discussed in Sec. IVA.

In addition to the return rate, we can also numerically obtain the Fisher zeros for a quench from the AFM into the LL phase. An example is shown in Fig. 11 where the initial state is a symmetric state. The lines of Fisher zeros now look much more complicated than for the quench in the transverse Ising case shown in Fig. 2, They form lines which seem to close for $R<0$. Furthermore, we find a number of "bifurcation points" where a line of zeros splits into two or where two lines join. In the considered case, the lines of Fisher zeros cross the $R=0$ axis at times $t_{c}$ where the return rate shows nonanalyticities. 




FIG. 11: (Color online) Fisher zeros for a quench from the AFM into the LL phase with $\Delta_{\text {ini }}=6$ and $\Delta_{\text {fin }}=0.8$. The lines of Fisher zeros cut the $R=0$ axis leading to nonanalyticities in the return rate $l(t)$. Lines are a guide to the eye.

\section{Quenches in the AFM phase}

Next, we consider quenches within the AFM phase. Because no phase transition line is crossed in this case, one might expect that the return rate is analytic. We will show, however, that this expectation is wrong in general. Our first example is presented in Fig. 12 where we show the return rate as well as next-leading eigenvalues of the transfer matrix for a quench where the initial state is either polarized are symmetric. In both cases, we clearly observe nonanalyticities in $l(t)$. While the return rate itself barely changes, the spectrum of the transfer matrix is different for the two initial states. The crossings between the leading and next-leading eigenvalue are clearly resolved numerically, in particular at later times, making this an obvious counterexample in the integrable case with regard to the hypothesis that cusps in $l(t)$ are always related to equilibrium quantum critical points.

Second, we show in Fig. 13 the lines of Fisher zeros for a different quench in the AFM phase. The structure of the Fisher zeros is again fairly complicated. Of particular interest is the line of Fisher zeros which approaches the $R=0$ axis at the longest simulation times. Here we finally cannot resolve whether or not this line will cut the $R=0$ axis at long times. What we do observe, however, is that the time where $l(t)$ shows the first cusp increases with decreasing $\Delta_{\text {ini }}$ while keeping $\Delta_{\text {fin }}$ fixed. This suggests that nonanalyticities are a generic feature for quenches within the AFM phase and that it is merely the limited simulation time which allows us to resolve cusps for the quench shown in Fig. 12, while we cannot resolve them for the case shown in Fig. 13.
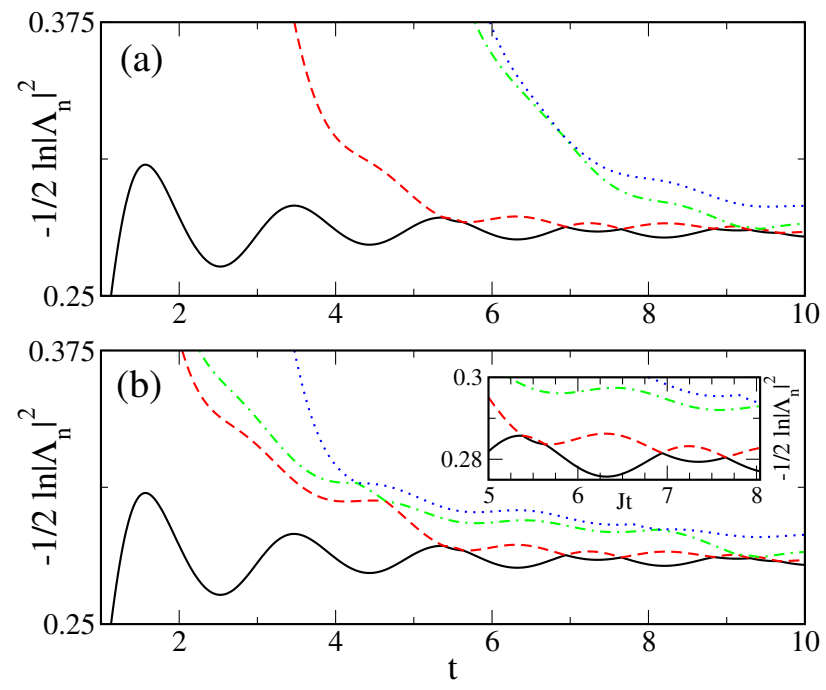

FIG. 12: (Color online) (a) Quench from the polarized initial state with $\Delta_{\text {ini }}=20000$ and $\Delta_{\text {fin }}=1.2$ which are both in the AFM phase. Contrary to what might have been expected, $l(t)$ shows cusps. (b) Same as in (a) for the symmetric initial state. All eigenvalues are twofold degenerate. The inset shows the regime where the first crossings of the leading and a nextleading eigenvalue occur.



FIG. 13: (Color online) Fisher zeros for a quench within the AFM phase with $\Delta_{\text {ini }}=6$ (symmetric) and $\Delta_{\text {fin }}=1.1$. The Fisher zeros might cut the $R=0$ axis at longer times. Lines are a guide to the eye.

\section{Quenches across the BKT transition driven by a staggered field}

Finally, we want to consider quenches where the initial state is the ground state of the nonintegrable Hamiltonian (III.1) with $h_{\text {st }} \neq 0$, while the time evolution is still integrable. For the quench shown in Fig. 14, we have chosen the parameters such that the critical line between the AFM and the LL phase is crossed, see the phase diagram in Fig. 3(b). As might be expected, the result looks 


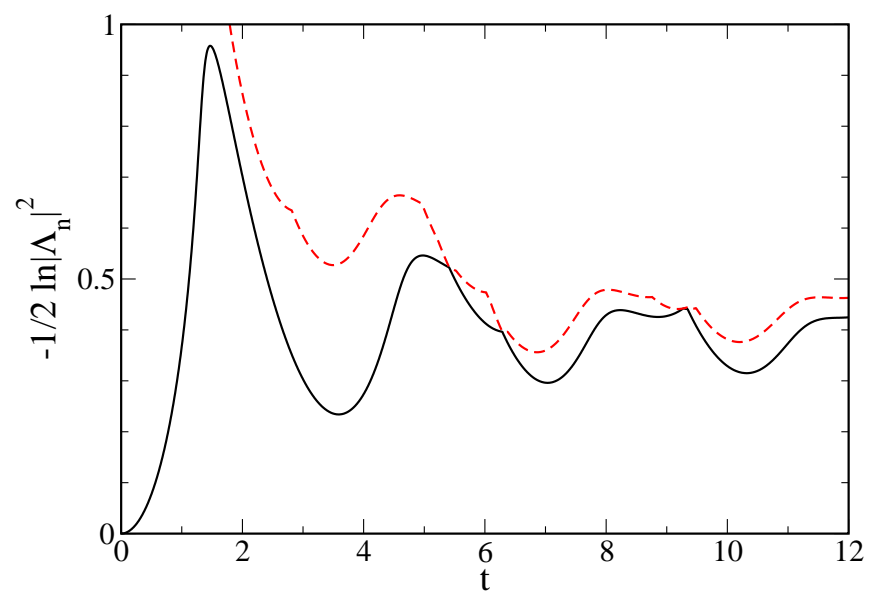

FIG. 14: (Color online) Return rate $l(t)$ and next-leading eigenvalues, $-\left(\ln \left|\Lambda_{n}\right|^{2}\right) / 2$, for a quench across the BKT transition from the AFM to the LL phase. Here $\Delta_{\text {ini }}=\Delta_{\text {fin }}=$ $-0.8, h_{\mathrm{st}, \text { ini }}=1.0$, and $h_{\mathrm{st}, \mathrm{fin}}=0.0$.

qualitatively similar to the quench from the AFM to the LL phase shown in Fig. 9 without the staggered field but with the initial state being polarized.

\section{B. Nonintegrable case}

Now we turn to quenches where the time-evolving Hamiltonian is given by (III.1) with $h_{\text {st }} \neq 0$. In this case the model is nonintegrable and the total magnetization and the Hamiltonian itself are the only local conserved charges. Subsystems of an infinite XXZ chain with staggered field are therefore expected to thermalize at long times after the quench.

\section{Quenches across the second-order transition driven by the staggered field}

For $\Delta>-1 / \sqrt{2}$, there is a second-order phase transition when changing the sign of the staggered field, see Fig. 3(b). The leading eigenvalues for a quench across this transition are shown exemplarily in Fig. 15 for a weakly interacting system. Nonanalyticities in $l(t)$ are present, however, the regions where crossings between the leading and the next-leading eigenvalue occur are very narrow in time so that it might be possible to find parameters where the second-order transition is crossed in the quench but $l(t)$ stays analytic.

By quenching the field instead from $h_{\text {st,ini }}=0.7$ to $h_{\mathrm{st}, \text { fin }}=-0.7$, we indeed no longer find any cusps in the numerically accessible time range, see Fig. 16. Obviously, we cannot rule out that nonanalyticities will show up at longer times. What we can say, however, is that apart from the counterexamples where a quantum phase transition is crossed but $l(t)=0$ discussed before -i.e., quenches from the FM phase or quenches where only $h$ is

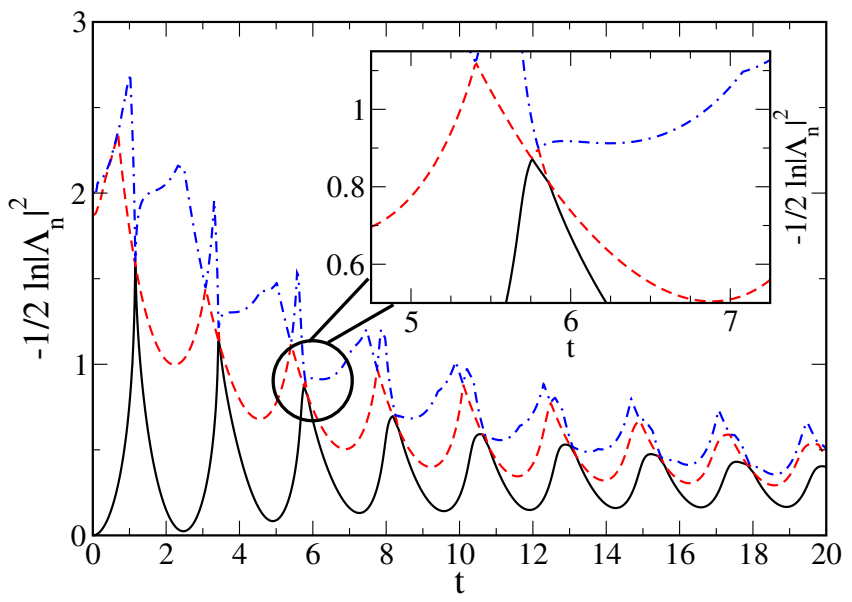

FIG. 15: (Color online) Return rate $l(t)$ (solid line) and nextleading eigenvalues, $-\left(\ln \left|\Lambda_{n}\right|^{2}\right) / 2$, for a quench across the second order transition between the AFM phases with $\Delta_{\text {ini }}=$ $\Delta_{\text {fin }}=0.1, h_{\text {st }, \text { ini }}=0.5$ and $h_{\text {st,fin }}=-0.5$.

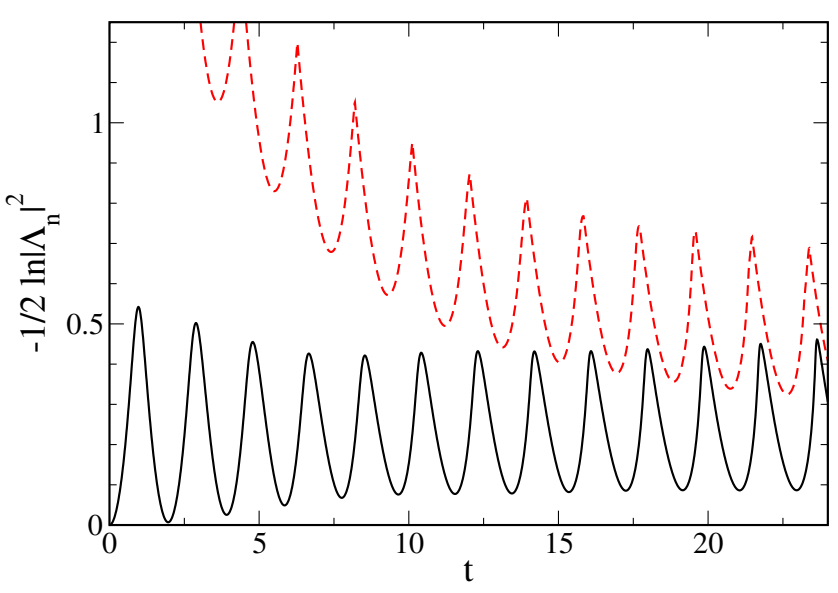

FIG. 16: (Color online) Return rate $l(t)$ (solid line) and nextleading eigenvalues, $-\left(\ln \left|\Lambda_{n}\right|^{2}\right) / 2$, for a quench across the second order transition between the AFM phases with $\Delta_{\text {ini }}=$ $\Delta_{\text {fin }}=0.1, h_{\text {st,ini }}=0.7$ and $h_{\text {st,fin }}=-0.7$.

changed - we also find quenches across equilibrium transitions leading to nontrivial dynamics but with $l(t)$ being analytic at least for very long times.

\section{Quenches across the BKT transition driven by a staggered field}

It is also possible to cross a BKT transition line by changing the staggered field. In Fig. 17, the return rate for such a quench is shown where we start with the same Luttinger liquid ground state as in Fig. 8 and quench into the AFM phase. Cusps in $l(t)$ are clearly visible. Here, the numerically accessible time range is again quite limited because of the slowly converging projection onto 


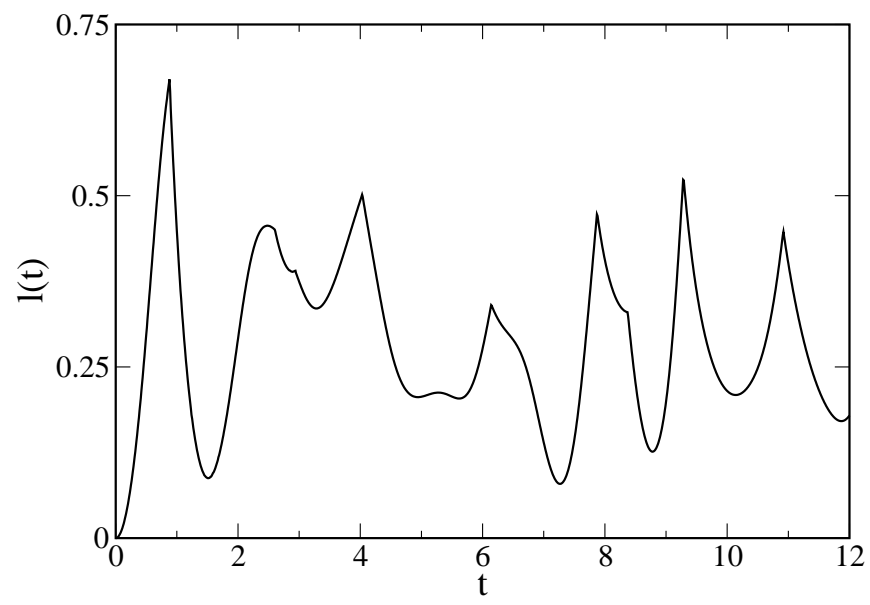

FIG. 17: (Color online) Return rate $l(t)$ for a quench across the BKT transition from the LL to the AFM phase. Here, $\Delta_{\text {ini }}=\Delta_{\text {fin }}=-0.8, h_{\mathrm{st}}^{\text {ini }}=0.0$, and $h_{\mathrm{st}}^{\mathrm{fin}}=1.0$.

the gapless ground state.

\section{Quenches within the AFM phase}

For the integrable model we discussed in Sec. VA 4 examples for quenches which do not cross an equilibrium phase transition but still show nonanalyticities in the return rate. Here we want to provide numerical evidence that such counterexamples also exist in the nonintegrable model. Our starting point are the two quenches in the integrable model from the AFM phase into the Luttinger liquid phase across the BKT point at $\Delta=1$ shown in Figs. 9 and 10. If we keep the same values for $\Delta_{\text {ini }}$ and $\Delta_{\text {fin }}$ and add a very small staggered field, we might expect that the return rate $l(t)$ will look very similar to the quench in Fig. 9 where we have used a polarized initial state. The quench puts a large amount of energy into the system and small changes to the low-energy spectrum will hardly matter. On the other hand, any arbitrarily small staggered field introduces a cosine term into the low-energy effective theory IV.15 which is relevant for $\Delta>-1 / \sqrt{2}$. Quenches where both $\Delta_{\text {ini }}$ and $\Delta_{\text {fin }}$ are larger than $-1 / \sqrt{2}$ therefore do not cross an equilibrium phase transition anymore. If nonanalyticities in $l(t)$ are always related to phase transitions, this would suggest that they do not occur for these kind of quenches.

From the numerical data in Fig. 18 it is obvious that the first reasoning is correct. Adding a small staggered field indeed hardly changes the return rate and Figs. 9 and 18 do indeed look very similar. In addition, we can calculate the lines of Fisher zeros, which are shown in Fig. 19. The line closest to the $t=0$ axis (the diagram is $t \rightarrow-t$ symmetric) closely approaches the $R=0$ axis but does not cross. At the time $t \approx 1.36$ where it does get closest to the $R=0$ axis, the return rate, shown in Fig. 18, has a sharp peak but remains analytic. The

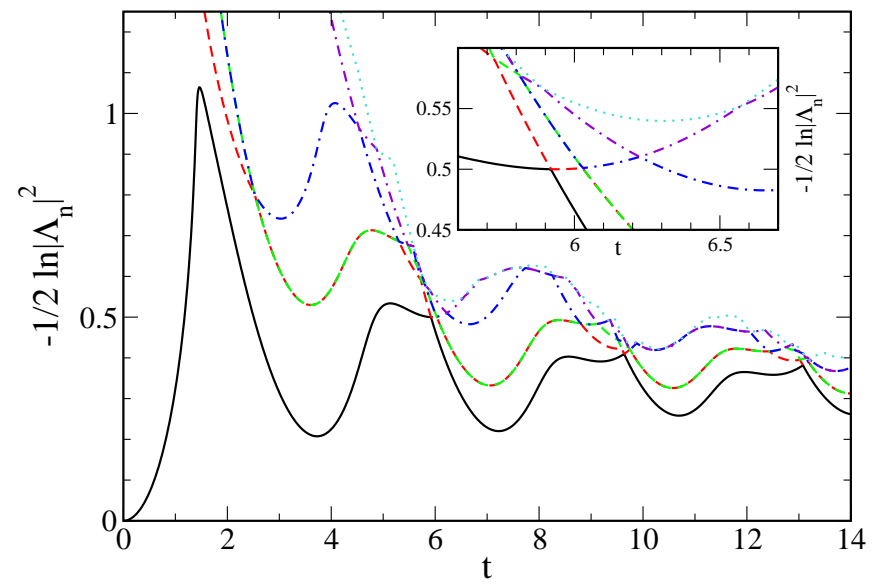

FIG. 18: (Color online) Return rate $l(t)$ and next-leading eigenvalues, $-\left(\ln \left|\Lambda_{n}\right|^{2}\right) / 2$, for a quench within the AFM phase. Here $\Delta_{\text {ini }}=6, \Delta_{\text {fin }}=-0.6$ while the staggered field is kept constant, $h_{\mathrm{st}}^{\mathrm{ini}}=h_{\mathrm{st}}^{\mathrm{fin}}=0.05$. Note that $l(t)$ shows nonanalyticities although no phase transition is crossed. The inset shows the regime where the first crossing occurs.



FIG. 19: (Color online) Fisher zeros for the same quench as in Fig. 18. The Fisher zeros cut the $R=0$ axis leading to the nonanalyticities in $l(t)$ shown in Fig. 18 Lines are a guide to the eye.

lines of Fisher zeros at larger times then do cross and the times where this happens are consistent with the nonanalyticities observed in $l(t)$ in Fig. 18. The considered case is therefore an example for a quench within the same phase of a generic quantum model where, nevertheless, the Fisher zeros do cross the $R=0$ axis.

\section{CONCLUSIONS}

The generalized Loschmidt amplitude $Z(z)=$ $\left\langle\Psi_{0} \mid \Psi(z)\right\rangle$ describes the overlap between an initial state $\left|\Psi_{0}\right\rangle$ and the evolved state $|\Psi(z)\rangle=\mathrm{e}^{-z H}\left|\Psi_{0}\right\rangle$ where $z$ is a complex number. For $z=\mathrm{i}$, this corresponds to 
real-time evolution, which is the experimentally accessible case. Similar to studies of the canonical partition function $Z_{\mathrm{th}}(z)=\operatorname{Tr} \mathrm{e}^{-z H}$ it is, however, interesting to study $Z(z)$ in the whole complex plane in order to understand the analytic structure. The so-called Fisher zeros of the partition function in the complex plane are important, because points where they approach the real axis in the thermodynamic limit determine the temperature where the corresponding thermodynamic potential becomes nonanalytic, indicating a phase transition. It therefore seems to be an excellent idea to carry this approach over to the Loschmidt echo and ask if dynamical phase transitions can be defined accordingly 11

For a $d$-dimensional quantum system, we can represent both $Z_{\text {th }}$ and $Z(z)$ as a lattice path integral of a $d+1$ dimensional classical model. The only difference is that the boundary conditions along the $z$ direction are periodic for $Z_{\mathrm{th}}$ while they are fixed by the initial state for $Z(z)$. For the classical system a transfer matrix can be defined in both cases that evolves along the spatial direction. The free energy $f(z)$ or the return rate, respectively, are then in the thermodynamic limit fully determined by the eigenvalue $\Lambda_{0}$ of the transfer matrix with largest modulus. Since eigenvalues can cross, nonanalyticities in these quantities are possible. In both cases the Fisher zeros are simply determined by the condition $\left|\Lambda_{0}\right|=\left|\Lambda_{n}\right|$ where $\Lambda_{n}$ is the next-leading eigenvalue with largest modulus, which is not degenerate with $\Lambda_{0}$ except at the crossing point.

We have described in this work a numerical algorithm to calculate the spectrum of the transfer matrix for $Z(z)$ in the one-dimensional case. By comparing with exact results for the transverse Ising model, we have shown that this algorithm can be used to precisely determine the Fisher zeros in the complex plane. We have then concentrated on the spin- $1 / 2 \mathrm{XXZ}$ model with a staggered magnetic field which we used to switch between integrable and nonintegrable dynamics. For a quench from a Néel state to the XX point (free fermions) we have analytically shown that the Fisher zeros sensitively depend on the initial state. While for a polarized Néel state as initial state, $\left|\Psi_{0}\right\rangle=|\uparrow \downarrow \uparrow \downarrow \cdots\rangle$, the Fisher zeros are just single points that lie on the $z=$ it axis, the Fisher zeros become closed, cigar-shaped lines in the complex plane if we use instead the symmetric state $\left|\Psi_{0}\right\rangle=(|\uparrow \downarrow \uparrow \downarrow \cdots\rangle+|\downarrow \uparrow \uparrow \uparrow \uparrow \cdots\rangle) / \sqrt{2}$. In addition, we used bosonization for quenches within the Luttinger liquid phase. The obtained result for the return rate $l(t)$, however, has to be considered with care. A sudden quench generally leads to particle-hole type excitations whose energies are only limited by the bandwidth of the microscopic model so that the dynamics is no longer dominated by low-energy excitations near the Fermi points. Only for very small quenches might the bosonization approach be justified. However, the bosonization result for $l(t)$ does even in this case depend on the ultraviolet cutoff which we had to use as a fitting parameter in our comparison with the numerical results. Allowing also for a small frequency shift, we did find reasonable agreement with the numerical data.

In the rest of the paper, we discussed numerical data for the Fisher zeros and return rates for various quenches in the XXZ with and without staggered field in cases where analytical results are not available. The main question we were trying to address is if nonanalyticities in the return rate following a sudden quench are always related to an equilibrium phase transition. For the XXZ model in the integrable and nonintegrable case, we pointed out two trivial counterexamples. (1) For any quench starting in the ferromagnetic phase, we have $l(t)=0$ because the ferromagnetic state is an eigenstate of the XXZ Hamiltonian for all parameters. (2) Any quench where only the uniform field $h$ is changed leads to $l(t)=0$ because the total magnetization always commutes with the Hamiltonian. In both cases, a crossing of an equilibrium phase transition in the quench does not leave any trace in $l(t)$. We also found indications that quenches in the staggered field across the second order transition between AFM phases might not lead to cusps in $l(t)$ for certain parameters or at least not in the numerically accessible time range.

Even more important, we also found examples where no phase transition is crossed but $l(t)$ nevertheless does show nonanalytic behavior. In the integrable XXZ model, such counterexamples are found for quenches within the antiferromagnetic phase from large values of anisotropy $\Delta$ to $\Delta \gtrsim 1$. In the nonintegrable model with staggered field, we did find such counterexamples again for quenches within the antiferromagnetic phase where the staggered field is kept constant and both the initial and the final values of anisotropy are larger than $-1 / \sqrt{2}$.

\section{Acknowledgments}

The authors acknowledge support by the Collaborative Research Centre SFB/TR49 and the Graduate School of Excellence MAINZ. We are grateful to the Regional Computing Center at the University of Kaiserslautern and the AHRP for providing computational resources and support.
1 W. H. Zurek, Rev. Mod. Phys. 75, 715 (2003).

2 D. P. DiVincenzo, Science 270, 255 (1995).

3 W. H. Zurek, Nature 412, 712 (2001).

${ }^{4}$ P. Jacquod, P. Silvestrov, and C. Beenakker, Phys. Rev. E
64, 055203 (2001).

5 R. A. Jalabert and H. M. Pastawski, Phys. Rev. Lett. 86, 2490 (2001).

6 A. Peres, Phys. Rev. A 30, 1610 (1984). 
7 T. Gorin, T. Prosen, T. H. Seligman, and M. Znidaric, Phys. Rep. 435, 33 (2006).

8 P. R. Levstein, G. Usaj, and H. M. Pastawski, J. Chem. Phys. 108, 2718 (1998).

9 H. Pastawski, P. Levstein, G. Usaj, J. Raya, and J. Hirschinger, Phys. A: Stat. Mech. and Appl. 283, 166 (2000).

10 H. T. Quan, Z. Song, X. F. Liu, P. Zanardi, and C. P. Sun, Phys. Rev. Lett. 96, 140604 (2006).

11 M. Heyl, A. Polkovnikov, and S. Kehrein, Phys. Rev. Lett. 110, 135704 (2013).

12 I. Bena, M. Droz, and A. Lipowski, Int. J. Mod. Phys. B 19, 4269 (2005).

13 T. D. Lee and C. N. Yang, Phys. Rev. 87, 410 (1952).

14 C. N. Yang and T. D. Lee, Phys. Rev. 87, 404 (1952).

15 S. Katsura and M. Ohminami, J. Phys. A 5, 95 (1972).

16 H. N. K. Yamada and M. Yamashita, Prog. Theor. Phys. 65, 1603 (1981).

17 M. E. Fisher, in Lectures in Theoretical Physics. Vol. VIIC / Statistical Physics, Weak Interactions, Field Theory (Univ. Colorado Press, Boulder, 1965).

18 W. van Saarloos and D. A. Kurtze, J. Phys. A 17, 1301 (1984).

19 C. Karrasch and D. Schuricht, Phys. Rev. B 87, 195104 (2013).

20 B. Pozsgay, arXiv:1308.3087 (2013).

21 M. Fagotti, arXiv:1308.0277 (2013).

22 M. Rigol, V. Dunjko, V. Yurovsky, and M. Olshanii, Phys. Rev. Lett. 98, 050405 (2007).

23 J. Sirker, N. P. Konstantinidis, F. Andraschko, and N. Sedlmayr, arXiv:1303.3064 (2013).

24 H. F. Trotter, Proc. Amer. Math. Soc. 10, 545 (1959).

25 M. Suzuki, Commun. Math. Phys. 51, 183 (1976).

26 M. Suzuki, Prog. Theor. Phys. 56, 1454 (1976).

27 A. Klümper, J. R. R. Martinez, C. Scheeren, and M. Shiroishi, J. Stat. Phys. 102, 937 (2001).

28 R. J. Bursill, T. Xiang, and G. A. Gehring, J. Phys. Cond. Mat. 8, L583 (1996).

29 X. Wang and T. Xiang, Phys. Rev. B 56, 5061 (1997).

${ }^{30}$ N. Shibata, J. Phys. Soc. Jpn. 66, 2221 (1997).
31 J. Sirker and A. Klümper, Europhys. Lett. 60, 262 (2002).

32 J. Sirker and A. Klümper, Phys. Rev. B 66, 245102 (2002).

33 J. Sirker and G. Khaliullin, Phys. Rev. B 67, 100408(R) (2003).

34 J. Sirker, Phys. Rev. B 69, 104428 (2004).

35 J. Sirker, J. Damerau, and A. Klümper, Phys. Rev. B 78, 235125 (2008).

36 S. Glocke, A. Klümper, and J. Sirker, Phys. Rev. B 76, 155121 (2007).

37 T. Enss and J. Sirker, New J. Phys. 14, 023008 (2012).

38 G. Vidal, Phys. Rev. Lett. 98, 070201 (2007).

39 U. Schollwöck, Rev. Mod. Phys. 77, 259 (2005).

40 M. Takahashi, Thermodynamics of one-dimensional solvable problems (Cambridge University Press, 1999).

41 F. C. Alcaraz and A. L. Malvezzi, J. Phys. A 28, 1521 (1995).

42 K. Okamoto and K. Nomura, J. Phys. A 29, 2279 (1996).

43 E. Lieb, T. Schultz, and D. Mattis, Annals of Physics 16, 407 (1961).

44 T. Giamarchi, Quantum physics in One Dimension (Clarendon Press, Oxford, 2004).

45 M. A. Cazalilla, Phys. Rev. Lett. 97, 156403 (2006).

46 R. Sachdeva, T. Nag, A. Agarwal, and A. Duta, arXiv:1311.1926 (2013).

47 B. Dóra, F. Pollmann, J. Fortágh, and G. Zaránd, Phys. Rev. Lett. 111, 046402 (2013).

48 M. Grabowski and P. Mathieu, Ann. Phys. 243, 299 (1995).

49 F. H. L. Essler, H. Frahm, F. Göhmann, A. Klümper, and V. E. Korepin, The One-Dimensional Hubbard model (Cambridge University Press, Cambridge, 2005).

50 T. Prosen, Phys. Rev. Lett. 106, 217206 (2011).

51 T. Prosen and E. Ilievski, Phys. Rev. Lett. 111, 057203 (2013).

52 M. Rigol, V. Dunjko, and M. Olshanii, Nature 452, 854 (2008).

53 M. Fagotti and F. H. L. Essler, Phys. Rev. B 87, 245107 (2013). 